\title{
Intra-Amygdala and Systemic Antagonism of NMDA Receptors Prevents the Reconsolidation of Drug-Associated Memory and Impairs Subsequently Both Novel and Previously Acquired Drug-Seeking Behaviors
}

\author{
Amy L. Milton, Jonathan L. C. Lee, Victoria J. Butler, Richard Gardner, and Barry J. Everitt \\ Behavioural and Clinical Neuroscience Institute, Department of Experimental Psychology, University of Cambridge, Downing Site, Cambridge CB2 3EB, \\ United Kingdom
}

\begin{abstract}
The amygdala has long been considered a primary locus in mediating the effects of previously drug-associated stimuli on subsequent drug-seeking behavior, and the NMDA subtype of glutamate receptor within the amygdala is important for the consolidation of associations between environmental conditioned stimuli and the effects of addictive drugs. Here we demonstrate that amygdala NMDA receptors are also necessary for the reconsolidation of drug-associated memories. Using a behavioral task that specifically measures the conditioned reinforcing properties of a previously drug-paired stimulus, we show that infusion of the NMDA receptor antagonist $\mathrm{D}(-)$ 2-amino-5-phosphonopentanoic acid (D-APV) into the basolateral amygdala before a memory reactivation session disrupted the drugassociated memory and abolished subsequent instrumental responding for conditioned reinforcement. This effect was memory reactivation dependent, and the memory deficit persisted for at least 4 weeks. In contrast, infusion of D-APV immediately after the memory reactivation session had no effect on subsequent responding for conditioned reinforcement, indicating that NMDA receptors have a temporally limited role in the reconsolidation process. Furthermore, in molecular studies, we show that the reconsolidation-impairing effect of D-APV is correlated with downstream reductions in expression of the plasticity-related immediate early gene, zif268. We also demonstrate that systemic antagonism of NMDA receptors with MK-801 [(+)-5-methyl-10,11-dihydro-SH-dibenzo[a,d]cyclohepten5,10-imine maleate] before memory reactivation subsequently reduced previously acquired instrumental drug-seeking behavior that depends on drug-associated cues acting as conditioned reinforcers. These data suggest that drugs modulating glutamatergic transmission at the NMDA receptor may be useful in the future treatment of relapse prevention in drug addiction through memory reconsolidation blockade.
\end{abstract}

Key words: memory reconsolidation; cocaine; self-administration; glutamate; NMDA receptor; zif268

\section{Introduction}

The impact on addictive behavior of associations between environmental conditioned stimuli (CSs) and self-administered drug, arising from repeated pairings, is central to several theories of addiction (Stewart et al., 1984; O’Brien et al., 1992; Robinson and Berridge, 1993; Everitt et al., 2001). Because memories elicited by exposure to drug CSs are a major contributor to relapse (Stewart et al., 1984; O’Brien et al., 1992; Everitt et al., 1999), treatments that disrupt drug-associated memories could act as a pro-abstinence or anti-relapse therapy (Lee et al., 2005, 2006a).

\footnotetext{
Received April 21, 2008; revised June 10, 2008; accepted June 27, 2008.

This work was supported by United Kingdom Medical Research Council (MRC) Grant 9536855 and was conducted within the MRC/Wellcome Trust Behavioural and Clinical Neuroscience Institute. A.L.M. was financially supported by an MRC research studentship, a studentship from Newnham College, Cambridge, and a research fellowship from Downing College, Cambridge, UK.

Correspondence should be addresssed to Amy L. Milton, Department of Experimental Psychology, University of Cambridge, Downing Site, Cambridge CB2 3EB, UK. E-mail: alm46@cam.ac.uk.

J. L. C. Lee's present address: School of Psychology, University of Birmingham, Birmingham B15 2TT, UK. DOI:10.1523/JNEUROSCI.1723-08.2008

Copyright $\odot 2008$ Society for Neuroscience $\quad 0270-6474 / 08 / 288230-08 \$ 15.00 / 0$
}

Memory reconsolidation, the process by which memories become destabilized by reactivation at retrieval and require restabilization to persist (Lewis, 1979; Nader, 2003), provides a key mechanism on which such treatments might act (Lee et al., 2005), and the NMDA subtype of glutamate receptor (NMDAR) is a promising target for drugs designed to disrupt memory reconsolidation.

The NMDAR has been recently implicated in memory reconsolidation. Antagonism of NMDARs has been shown to be amnestic when given in conjunction with memory retrieval, suggesting their involvement in the reconsolidation of spatial memories (Przybyslawski and Sara, 1997), sucrose-associated memories (Lee and Everitt, 2008), fear-associated memories (Ben Mamou et al., 2006; Lee et al., 2006b), and drug-conditioned place preference memories (Kelley et al., 2007; Sadler et al., 2007). Furthermore, antagonism at the NMDAR has been shown to reduce the expression of the plasticity-related immediate early gene zif268 during classical conditioning (Mokin and Keifer, 2005), which is also increased on the retrieval of drug-associated memories (Thomas et al., 2003). The knockdown of zif268 within the baso- 
lateral amygdala (BLA) also impairs drug-associated memory reconsolidation (Lee et al., 2005). Therefore, in addition to investigating the behavioral effects of NMDAR antagonism at memory retrieval on drug-associated memories, we also investigated the effects of NMDAR antagonism on the downstream expression of zif268.

The neural site at which systemic NMDAR antagonism disrupts drug-associated memory reconsolidation has not yet been defined, but the BLA is likely to be a primary locus. The BLA mediates conditioned reinforcement, because it has been shown to be critical for the mechanism by which drug-associated stimuli affect instrumental behavior (Cador et al., 1989; Burns et al., 1993), including cue-induced reinstatement of cocaine-seeking behavior in models of relapse (Meil and See, 1997; Kantak et al., 2002). Therefore, in the study reported here, we have used both intra-BLA and systemic NMDAR antagonism in two models of responding for drug-associated conditioned reinforcers (Di Ciano and Everitt, 2004; Lee et al., 2005, 2006a; Lu et al., 2005) to investigate the reconsolidation of CS-cocaine memories, and especially whether the BLA is a primary locus of the amnestic effect of systemically administered NMDAR antagonists. We also measured the expression of zif268 to investigate the cellular correlates of the behavioral effects of intra-BLA NMDAR antagonism.

\section{Materials and Methods \\ Subjects}

Subjects were male Lister Hooded rats (Charles River) weighing at least $250 \mathrm{~g}$ at the time of surgery. Each animal was housed singly after surgery in a vivarium on a reverse light/dark cycle (lights on at 7:00 P.M.). Subjects were food restricted, being fed after training or testing, and allowed access to water ad libitum except during training and testing sessions. All experiments were conducted in accordance with the United Kingdom Animals (Scientific Procedures) Act of 1986 (PPL 80/1767).

\section{Surgery}

Rats were implanted with a chronic, indwelling intravenous catheter targeting the right jugular vein and, for experiment 1, bilateral cannulas located just dorsal to the basolateral amygdala. The coordinates for cannula implantation were anteroposterior $-2.6 \mathrm{~mm}$ and mediolateral $\pm 4.5 \mathrm{~mm}$ (relative to bregma), and dorsoventral $-5.6 \mathrm{~mm}$ (relative to dura). Details of the surgical procedures can be found in the article by Lee et al. (2005). A recovery period of $7 \mathrm{~d}$ was imposed before behavioral training and testing began.

\section{Infusions}

Infusions were performed using a syringe pump and $5 \mu$ l Hamilton syringes, connected to injectors (28 gauge, projecting $2 \mathrm{~mm}$ beyond the guide cannulas) by polyethylene tubing. Infusions of the competitive NMDAR antagonist D(-)-2-amino-5-phosphonopentanoic acid (DAPV) $(5 \mu \mathrm{g} / 0.5 \mu \mathrm{l} / \mathrm{side}, 0.25 \mu \mathrm{l} / \mathrm{min}$; Sigma-Aldrich $)$ or sterile PBS vehicle $(0.5 \mu \mathrm{l} / \mathrm{side}, 0.25 \mu \mathrm{l} / \mathrm{min})$ were begun $30 \mathrm{~s}$ after the insertion of the injectors and performed over $2 \mathrm{~min}$. One minute of waiting time was imposed from the end of the infusion to the removal of injectors to allow diffusion of the solution away from the infusion site. This dose, administered directly to the BLA, has been shown to disrupt the acquisition of conditioned fear memory (Matus-Amat et al., 2007). Rats were habituated to the infusion procedure using the vehicle solution $(0.5 \mu \mathrm{l} / \mathrm{side}$, $0.25 \mu \mathrm{l} / \mathrm{min}$ ) twice during self-administration training, always before conditioning.

\section{Injections}

Because D-APV poorly penetrates the CNS when administered systemically, an alternative NMDAR antagonist, (+)-5-methyl-10,11-dihydro$\mathrm{SH}$-dibenzo[a,d]cyclohepten-5,10-imine maleate (MK-801), was used for systemic administration. Rats in the reactivated groups were given intraperitoneal injections of MK-801 (0.1 mg/kg; Sigma-Aldrich) or an equivalent volume of $0.9 \%$ saline vehicle $30 \mathrm{~min}$ before the memory reactivation session. Non-reactivated groups were injected on the same day and were immediately returned to their home cages without experiencing the memory reactivation session. This dose of MK- 801 has been demonstrated previously to lead to deficits in the reconsolidation of aversive and appetitive memories (Lee et al., 2006b; Lee and Everitt, 2008).

\section{Behavioral procedures}

Experiment 1a: acquisition of a new instrumental response for a previously drug-paired conditioned reinforcer. Rats were trained in operant chambers (Med Associates) as described previously (Hellemans et al., 2006). Selfadministration training took place over nine daily sessions, controlled by the Whisker control system. Each head entry (nosepoke) into the food magazine was recorded and reinforced on a fixed-ratio 1 (FR1) schedule with a presentation of a $20 \mathrm{~s}$ light CS and a $5.83 \mathrm{~s}$ infusion of cocaine $(0.25$ $\mathrm{mg} / 0.1 \mathrm{ml}$ ), during which time the houselight was not illuminated. Nosepokes made during the $20 \mathrm{~s}$ of CS presentation were not reinforced. The end of this time-out period was signaled by the illumination of the houselight. The rats were permitted a maximum of 30 cocaine infusions, after which the houselight was turned off and the session ended. If the rats did not reach this limit, the session terminated $60 \mathrm{~min}$ after it started.

Memory reactivation took place on the day after the final session of self-administration training. For the infusions of D-APV given before memory reactivation, the reactivation session began immediately after the end of the infusion procedure. Rats receiving postreactivation (PR) infusions were infused immediately after the end of the reactivation session. In the reactivation session, all of the parameters were the same as during training, except that the session length was $15 \mathrm{~min}$, and the syringe pump delivered saline instead of cocaine. Non-reactivated controls were given intracerebral infusions and returned to the home cage.

Testing for conditioned reinforcement by measuring the acquisition of a new instrumental response reinforced by the CS (ANR) began the day after the memory reactivation session. Rats were returned to the same operant chamber that had previously been used for training and reactivation, but this now contained two novel levers. Depression of the inactive lever, which was located on the same side as the CS light to avoid the confound of pavlovian approach behavior (Parkinson et al., 2005), produced no programmed consequence but was recorded for comparison to active lever presses. Active lever presses produced a 1 sillumination of the previously drug-paired light CS on a variable ratio 1-3 schedule, during which time the houselight was switched off. The abbreviated CS was used because this is optimal for the CS to act as a conditioned reinforcer (Mackintosh, 1974). There was no limit to the number of CS presentations during ANR; the rats remained in the chamber for $30 \mathrm{~min}$. ANR testing was conducted on PR days 1, 2, 5, and 8 and, if an impairment was evident, on PR days 15, 22, and 29.

Experiment 1b: analysis of Zif268 expression after antagonism at the NMDAR. Six groups of rats underwent the same surgery and behavioral training procedures as described for experiment 1a, but they were killed $2 \mathrm{~h}$ after the intracerebral infusions for assessment of Zif268 protein levels in the basolateral amygdala. All infusion parameters and, where applicable, the parameters of the reactivation session, were the same as in experiment 1a. After killing by exposure to a rising $\mathrm{CO}_{2}$ concentration, and decapitation, the brains were rapidly extracted and immediately frozen on dry ice.

Experiment 1c: conditioned place aversion. To ensure that any reduction in responding for the previously drug-paired CS was attributable to a deficit in CS-drug memory reconsolidation and not counterconditioning of any putative aversive effects of NMDAR antagonism to the previously drug-paired CS, the ability of the D-APV infusion to act as an unconditioned stimulus in the conditioning of a place aversion was investigated.

Before each session, the two-compartment conditioned place aversion apparatus was cleaned with TriGene spray to remove any olfactory cues from previous sessions. The rats always began the sessions in the central compartment of the chamber, before being released into the appropriate chamber(s). On the day before conditioning, the rats were habituated to the apparatus for $20 \mathrm{~min}$, with the time spent in each compartment measured by the experimenter on a video link in an adjacent room. On the conditioning day, the rats were confined in one of the compartments 
for $20 \mathrm{~min}$ immediately after an intra-BLA infusion of D-APV $(0.5 \mu \mathrm{g} / 0.5 \mu \mathrm{l}, 0.25 \mu \mathrm{l} / \mathrm{min})$. The conditioned side was arbitrarily assigned to each rat, with the groups being matched so that times spent in the to-be-conditioned compartment during the habituation session were equal. On the following day, the rats' preferences for each compartment were tested by reexposing them to the apparatus for $20 \mathrm{~min}$, and recording the time spent in each compartment.

Experiment 2: maintenance of cocaine-seeking with conditioned reinforcement. Rats were trained in operant conditioning chambers (Med Associates) as described previously (Lee et al., 2006a). Rats underwent $10 \mathrm{~d}$ of cocaine self-administration training in which depression of the "active" lever (counterbalanced left or right) led to a cocaine infusion $(0.25 \mathrm{mg} / 0.1$ $\mathrm{ml}$ delivered over $5.83 \mathrm{~s}$ ) and a $20 \mathrm{~s}$ illumination of the CS-light, which was located above the active lever on a FR1 schedule of reinforcement. Responding on a second lever, the "inactive" lever, although recorded, had no programmed consequences. The rats were limited to $30 \mathrm{CS}$-cocaine pairings in a session. If this limit was not reached, the session terminated $60 \mathrm{~min}$ after it started.

Memory reactivation took place $2 \mathrm{~d}$ after the completion of cocaine self-administration training. The rats were returned to the operant conditioning chambers used during training for a 30 min reactivation session. The parameters of this session were the same as for training, except that depression of the active lever led to a $0.1 \mathrm{ml}$ infusion of saline, rather than cocaine, paired with the $20 \mathrm{~s}$ CS-light. Non-reactivated control groups received injections and were immediately returned to the home cage.

Testing for cocaine seeking with conditioned reinforcement occurred $6 \mathrm{~d}$ after the completion of self-administration training. The rats were returned to the operant conditioning chambers used for training with both levers presented. Depression of the active lever led to the presentation of an abbreviated $1 \mathrm{~s}$ light CS and lever retraction. Pressing of the inactive lever had no programmed consequence, but was recorded to give an indication of generalized activity. There was no limit to the number of CS presentations during the test, the session being terminated $60 \mathrm{~min}$ after it had started. The data from the test session were analyzed in four, 15 min time bins.

\section{Histological assessment of cannula placements (experiments 1 a and 1c)}

After the end of testing, rats with intracerebral cannulas that were not used for experiment $1 \mathrm{~b}$ were killed with an overdose of sodium pentobarbital [ $1.5 \mathrm{ml}$ of Dolethal (Rhone Merieux) per animal] and perfused with 0.01 м PBS followed by $4 \%$ paraformaldehyde. Coronal sections at $60 \mu \mathrm{m}$ were stained with cresyl violet so that the placements could be verified by light microscopy. Subjects were only included if there was evidence of the injector tip being placed within the basolateral amygdala, and there was no bilateral damage to the BLA or other areas of the brain. The cannula placements in the animals in experiment $1 \mathrm{~b}$ were verified by eye before amygdala dissection.

\section{Western blotting (experiment $1 b$ )}

After being killed, the rats were decapitated, and the brains were extracted and frozen on dry ice before being stored at $-80^{\circ} \mathrm{C}$. After mild thawing, the cannula placements were verified by eye, and $1 \mathrm{~mm}$ sections were taken using an adjustable brain matrix (Braintree Scientific). The amygdala was dissected bilaterally using a $1 \mathrm{~mm}$ brain punch set (Stoelting), and the tissue lysates and Western blotting were performed as described previously (Lee et al., 2004). The proteins were separated on $7.5 \%$ Tris- $\mathrm{HCl}$ gels using a constant voltage of $80 \mathrm{~V}$ and then transferred to nitrocellulose membranes at a constant current of $100 \mathrm{~mA}$ for $1 \mathrm{~h}$. The blots were blocked in a $0.01 \mathrm{~m}$ Tris-buffered saline solution containing
$0.05 \%$ Tween 20 (TBST) that also contained 5\% nonfat milk. The TBST solution was used for all subsequent incubations and washes. The blots were first incubated in primary antibodies [Zif268, 1:10,000 (a kind gift from G. I. Evan, University of California, San Francisco, San Francisco, CA); $\beta$-actin, 1:20,000] and then secondary antibodies [goat antirabbit and goat anti-mouse IgG (whole-molecule)-peroxidase conjugates, 1:10,000]. The blots were developed by enhanced chemiluminescence and opposing to autoradiographic film. Images of these autoradiographs were captured using a Qicam 12-bit mono digital camera (QImaging) and quantified using MCID Core 7.0 software (InterFocus Imaging). Autoradiographs were developed so as to be linear in the range used to analyze the density of $\beta$-actin and Zif268. For each sample, the amount of protein was analyzed by measuring the optical density and the area of the protein band, and these were multiplied to derive a value for the amount of protein for both $\beta$-actin and Zif268. Although the amount of protein in each sample was normalized before Western blotting using a NanoDrop ND-1000 spectrophotometer (LabTech International), any variation in loading was corrected by deriving a normalization factor (average amount of $\beta$-actin/sample amount of $\beta$-actin) for each sample. This factor was then used to correct the raw sample amount of Zif268, which was finally normalized against the average control level of Zif268 expression in the vehicle group.

\section{Statistical analysis}

All data were analyzed using SPSS 11.5 for Windows (SPSS). Unless otherwise stated, the data were analyzed using a three-way repeated measures ANOVA, with Lever and Session as within-subject factors, and Treatment (D-APV vs PBS vehicle) or Group (vehicle, CS present vs MK-801, CS present vs vehicle, CS omitted) and Reactivation (reactivated vs non-reactivated) as the between-subject factors. Any deviations from sphericity were corrected using the Greenhouse-Geisser correction if $\varepsilon<0.75$, and the Huynh-Feldt correction if $\varepsilon>0.75$, as recommended by Cardinal and Aitken (2006). Where appropriate, Sidak-corrected pairwise comparisons were performed; the Sidak correction is the mathematically correct version of the Bonferroni correction (Cardinal and Aitken, 2006). Student's $t$ tests (two-tailed) were used to compare means of the experimental groups in the memory reactivation session, and the molecular data were analyzed using one-tailed $t$ tests, because we specifically predicted a reduction in Zif268 expression levels in the group receiving D-APV treatment before memory reactivation.

\section{Results}

Experiment 1a: acquisition of a new response for a previously drug-paired conditioned reinforcer

All of the cannula placements were within or adjacent to the boundaries of the BLA. Only rats with patent cannulas that were 
a
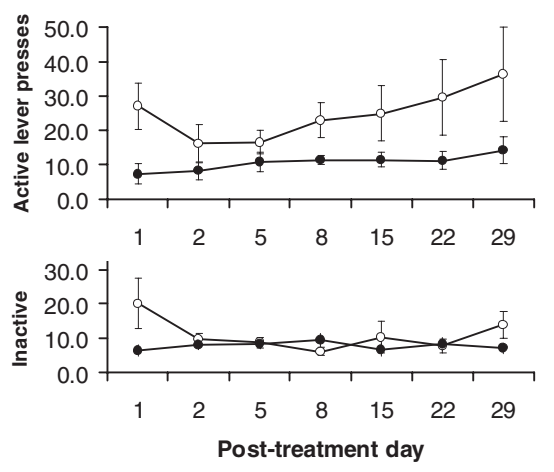

$\rightarrow$ Vehicle $\rightarrow$ D-APV b
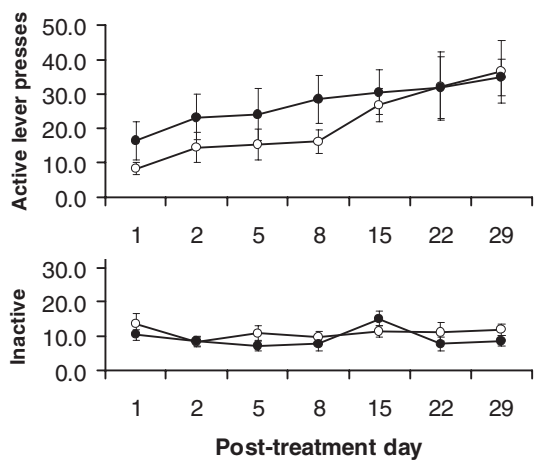

$\rightarrow$ Vehicle -- D-APV
C
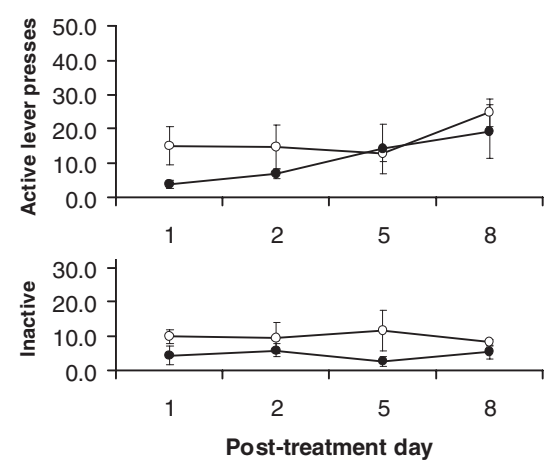

$\multimap$ Vehicle $\rightarrow$ D-APV

Figure 2. Assessment of the conditioned reinforcing properties of a previously cocaine-paired CS using the acquisition of a new instrumental response for conditioned reinforcement. $\boldsymbol{a}$, The number of lever presses made for the conditioned reinforcer (active lever) and for no reinforcement (inactive lever) $>29 \mathrm{~d}$ after treatment by rats infused with $\mathrm{D}$-APV or PBS vehicle before the memory reactivation session (vehicle, $n=5 ; \mathrm{D}-\mathrm{APV}, n=7)$. $\boldsymbol{b}$, The number of lever presses made for conditioned reinforcement by rats infused but not reactivated (vehicle, $n=9 ; \mathrm{D}-\mathrm{APV}, n=7$ ). $c$, The number of lever presses made for conditioned reinforcement by rats infused immediately after the memory reactivation session ( $n=4$ per group). Data are presented as mean \pm SEM.

placed either within or adjacent to the boundaries of the basolateral amygdala were included in the analysis (Fig. 1).

Intra-amygdala NMDAR antagonism at memory reactivation persistently disrupted the ability of the previously drug-paired CS to act as a conditioned reinforcer, in a reactivation-dependent manner

Treatment with the NMDA receptor antagonist D-APV immediately before the memory reactivation session led to a subsequent persistent impairment in the conditioned reinforcing properties of the previously drug-paired CS (Fig. 2a). The rats administered D-APV before memory reactivation did not discriminate between the lever that produced the previously drug-paired CS (active lever) and the lever that had no programmed consequences (inactive lever), in contrast to the vehicle-infused, reactivated control group (Sidak-corrected pairwise comparisons, vehicle: $p<$ 0.01; D-APV: $p=0.356$ ). The deficit induced by D-APV was reactivation dependent (treatment $\times$ reactivation $\times$ lever: $F_{(1,24)}=$ $4.849, p<0.05)$, because although there was a main effect of treatment with the NMDAR antagonist in the reactivated groups (treatment: $F_{(1,10)}=6.488, p<0.05$; lever $\times$ treatment: $F_{(1,10)}=$ $5.269, p<0.05)$, there was no such difference in the nonreactivated condition (treatment: $F<1$; lever: $F_{(1,14)}=20.878$, $p<0.001$; lever $\times$ treatment: $\left.F_{(1,14)}=1.405, p=0.256\right)($ Fig. $2 b)$. Sidak-corrected pairwise comparisons supported the deficit in lever pressing seen in the reactivated, D-APV-treated group as being specifically related to the presentation of the previously drug-associated cue, because the reactivated vehicle-treated group pressed the active lever more than did the reactivated D-APV-treated group ( $p=0.028)$, whereas both treatment groups showed the same levels of inactive lever pressing ( $p=$ $0.142)$.

Postmemory reactivation NMDAR antagonism had no effect on the acquisition of responding with conditioned reinforcement Infusions of D-APV immediately after the memory reactivation session did not impair subsequent instrumental responding for conditioned reinforcement (Fig. 2c). Because there were no significant differences between the groups by day 8 after reactivation, subsequent testing was not conducted. All of the rats, both vehicle- and D-APV-treated, showed a preference for the active lever over the inactive lever (lever: $F_{(1,6)}=8.148, p<0.05$; treat-

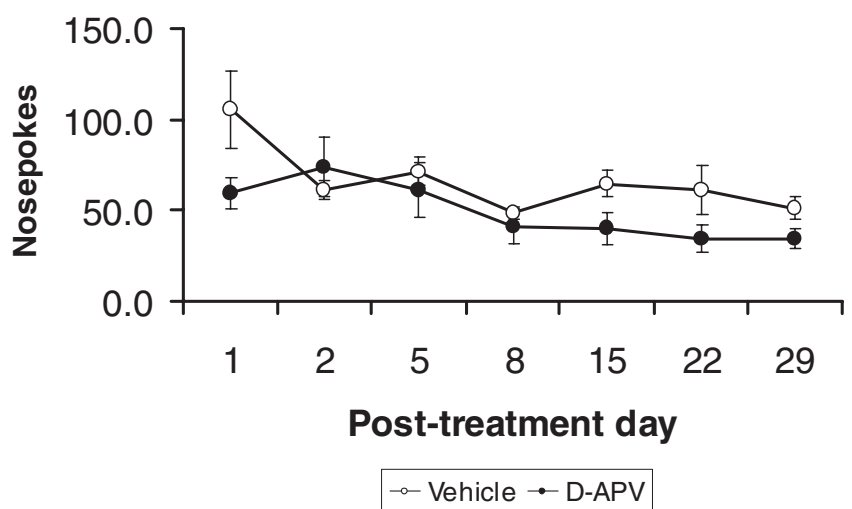

Figure 3. Performance of the previously drug-reinforced instrumental behavior, nosepoking, during subsequent testing for conditioned reinforcement was not affected by the administration of D-APV before memory reactivation (vehicle, $n=5$; D-APV, $n=7$ ). Data are presented as mean \pm SEM.

ment: $F_{(1,6)}=2.934$, n.s.; lever $\times$ session: $F_{(3,18)}=3.246, p<$ 0.05; lever $\times$ treatment: $F<1$ ).

Subsequent performance of the previously learned instrumental response for drug was not reduced by NMDAR antagonism before memory reactivation

Because a deficit in responding for subsequent conditioned reinforcement was found in the experimental group given D-APV in conjunction with memory reactivation, the primary instrumental response that had previously been reinforced with drug, nosepoking, was also analyzed for the experimental groups that received infusions before the memory reactivation session. However, in contrast to the deficit observed with the acquisition of a new instrumental response with conditioned reinforcement, $\mathrm{D}-\mathrm{APV}$ given in conjunction with memory reactivation did not reduce the number of nosepokes, the previously acquired instrumental behavior, relative to the vehicle-infused control group, during the subsequent test phase (Fig. 3) (treatment: $F_{(1,10)}=$ 2.624 , n.s.; session $\times$ treatment: $F_{(2.34,23.4)}=2.089$, n.s. $)$. However, nosepoking did extinguish throughout the course of testing (session, $\left.F_{(2.34,23.4)}=5.324, p<0.01\right)$. 

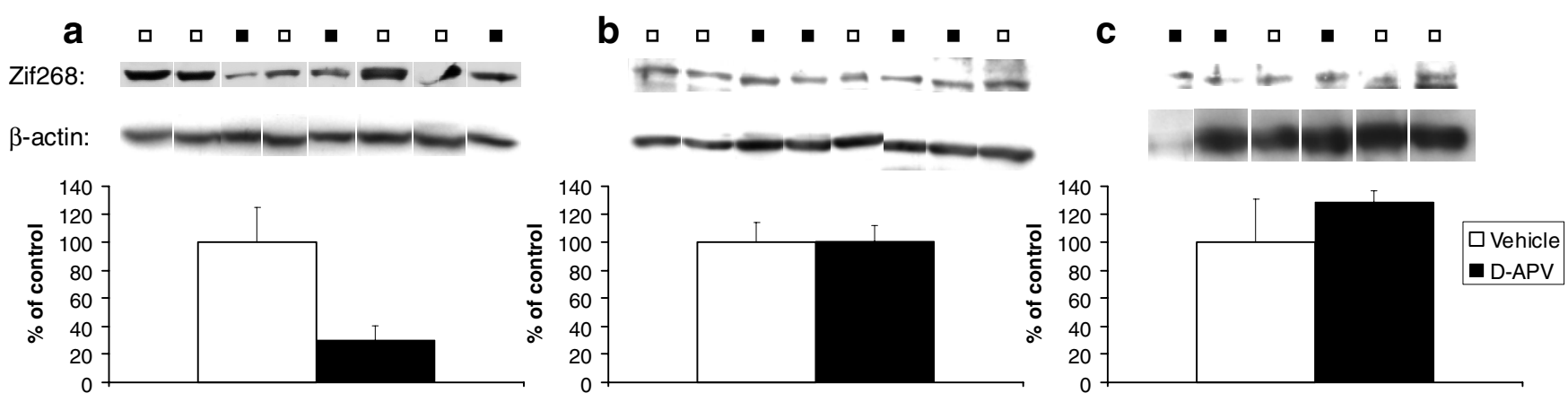

Figure 4. Analysis of Zif268 expression levels using Western blots. Representative Western blots are shown above bar charts, quantifying levels of Zif268 and $\beta$-actin expression relative to controls. White squares represent vehicle-infused animals; black squares represent D-APV-infused animals. $\boldsymbol{a}$, Administration of the NMDAR antagonist D-APV immediately before memory reactivation led to a downregulation of Zif268 (vehicle, $n=5 ; \mathrm{D}-\mathrm{APV}, n=3$ ). $\boldsymbol{b}, \boldsymbol{c}$, Zif268 expression levels were not affected when D-APV was administered in the absence of a memory reactivation session ( $n=4$ per group) (b), or when D-APV was administered immediately after the memory reactivation session ( $n=3$ per group) (c). Data are presented as mean + SEM.

There were no differences in cocaine self-administration between the experimental groups

There were no differences in training performance between any of the experimental groups and their vehicle controls (data not shown). There were no differences between the experimental groups that received pre-reactivation infusions and those that were non-reactivated in terms of either CS-cocaine pairings experienced during training (treatment: $F<1$; reactivation: $F<1$; treatment $\times$ reactivation: $F<1$ ) or the number of instrumental responses (nosepokes) made for cocaine during selfadministration training (treatment: $F<1$; reactivation: $F<1$; treatment $\times$ reactivation: $F_{(1,24)}=1.317$, n.s.).

Training performance was equivalent between the vehicle and D-APV experimental groups to be given the infusion immediately after memory reactivation, with no differences in CS-cocaine pairings experienced during self-administration (treatment: $F<$ 1; session $\times$ treatment: $F<1$ ) or nosepokes made for cocaine (treatment: $F_{(1,6)}=1.692$, n.s.; session $\times$ treatment: $F<1$ ).

Because the amount of CS exposure has been suggested to influence the degree of memory reactivation (Suzuki et al., 2004), it was important to verify that there were no differences in the number of CS presentations experienced during the memory reactivation session. Thus, the mean number of nosepoke-CS presentations experienced by each experimental group was compared with the appropriate control group using independent samples $t$ tests. There were no differences in CS presentations for the reactivated groups given infusions before memory reactivation (data not shown; vehicle, $17.2 \pm 1.66$, D-APV, $14.0 \pm 2.73$, $t_{(10)}=0.90$, n.s.) or for the groups infused after the memory reactivation session (data not shown; vehicle, $12.5 \pm 2.75$, D-APV, $12.5 \pm 3.07, t_{(6)}=0.43$, n.s.).

\section{Experiment 1b: Zif268 expression levels after}

\section{NMDAR antagonism}

Intra-amygdala $D-A P V$ infused before memory reactivation reduced Zif268 expression, whereas D-APV administered without memory reactivation did not

Based on the findings from experiment $1 \mathrm{a}$ and our previous data showing a reduction in conditioned reinforcement with knockdown of the immediate early gene zif268 (Lee et al., 2005), we predicted that NMDAR antagonism was disrupting memory reconsolidation through downregulation of downstream zif268 expression. The molecular data supported these predictions, because D-APV infused immediately before memory reactivation reduced the levels of Zif268 expression measured $2 \mathrm{~h}$ after the infusion (treatment: $t_{(6)}=2.094, p<0.05$ ), whereas D-APV ad- ministered without a memory reactivation session had no effect (treatment: $t_{(6)}=0.044$, n.s.) (Fig. $4 a, b$ ). In contrast, postreactivation infusion of $\mathrm{D}-\mathrm{APV}$ had no effect on the subsequent levels of Zif268 expression (treatment: $t_{(2.1)}=1.012$, n.s.) (Fig. $4 c$ ), which is consistent with the behavioral finding that rats infused with D-APV after memory reactivation showed no subsequent reduction in the conditioned reinforcing properties of the previously drug-paired CS (Fig. $2 c$ ).

There were no differences in cocaine self-administration between the experimental groups

There were no differences in the training experience between any of the test and control groups. All of the experimental groups learnt the association between cocaine and the CS during training, because there were no differences in their training performance, either in terms of the number of CS-cocaine pairings experienced during training (data not shown; treatment: $F<1$ for pre-reactivation infused groups, non-reactivated groups, and post-reactivation infused groups) or in the number of nosepokes made for cocaine (data not shown; treatment: $F<1$ for prereactivation infused group and postreactivation infused group, $F_{(1,6)}=2.249$, n.s. for the non-reactivated group).

There were also no differences in the number of CS presentations experienced during the memory reactivation session for those groups that experienced it [data not shown; infusions before memory reactivation: vehicle, $17.0 \pm 2.74$, D-APV, $20.0 \pm$ $1.15, t_{(6)}=0.801$, n.s.; infusions after memory reactivation: vehicle, $18.0 \pm 0.91$, D-APV, $21.3 \pm 1.20, t_{(5)}=2.259$, n.s. $]$.

\section{Experiment 1c: intra-BLA NMDAR antagonism does not induce an aversive state}

Neither infusion of PBS nor infusion of D-APV produced aversive effects sufficient to condition a place aversion (Fig. 5). The time spent on the paired side was the same at test after the conditioning session as it was in the habituation session, before conditioning, and there were no differences between the rats infused with PBS and those infused with D-APV (treatment: $F<1$; session $X$ treatment: $F<1$; session: $F<1$ ).

Experiment 2: performance of a previously acquired cocaineseeking instrumental response with conditioned reinforcement

Overall analysis of the $1 \mathrm{~h}$ test session revealed that there was a pronounced within-session extinction of the effect of the previously drug-paired CS on responding that may have resulted in a floor effect in the vehicle group, reducing statistical power 


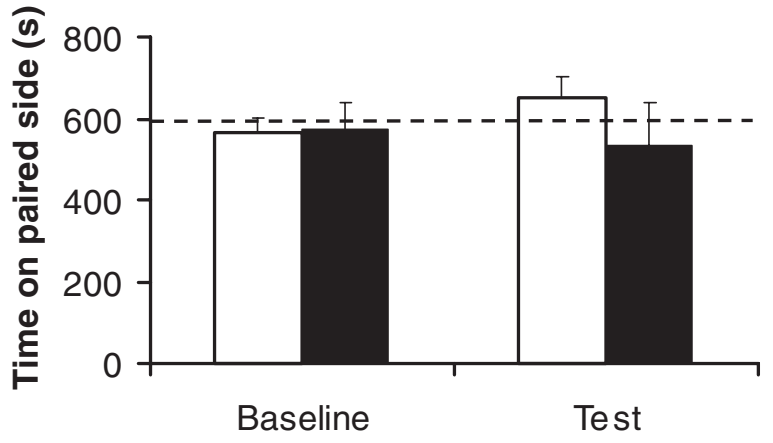

Figure 5. Time spent on the infusion-paired side of a two-compartment apparatus $1 \mathrm{~d}$ before the conditioning session (Baseline) and $1 \mathrm{~d}$ after the conditioning session (Test). The dotted line represents equal amounts of time being spent on the paired and unpaired sides (vehicle, $n=4 ; \mathrm{D}-\mathrm{APV}, n=5$ ). Data are presented as mean + SEM.

(bin: $F_{(2.69,69.8)}=30.013, p<0.001$; drug: $F<1$; drug $\times$ reactivation: $F=2.935, p=0.099)$. Therefore, the first 15 -min time bin was analyzed separately. MK-801, given before memory reactivation, reduced cue-maintained cocaine-seeking $3 \mathrm{~d}$ later (Fig. $6 a)$ but MK-801 injection without a memory reactivation session was insufficient to impair lever pressing (Fig. 6b) (treatment $X$ reactivation: $\left.F_{(1,26)}=5.044, p<0.05\right)$. Consistent with this, there was no main effect of Treatment in the non-reactivated groups (treatment: $F_{(1,10)}=1.045$, n.s.). However, whereas MK-801 administered at memory reactivation impaired responding relative to saline-treated rats (vehicle vs MK-801 reactivated groups, treatment: $\left.F_{(1,16)}=5.319, p<0.05\right)$, there was no difference in lever pressing compared with a reactivated, vehicle-treated control group in which the CS was omitted at test (MK-801 vs CSomission group, group: $F_{(1,16)}=3.287$, n.s.; lever $\times$ group: $F_{(1,16)}$ $=4.236$, n.s.). Solely comparing responding on the active levers, the rats that received MK-801 at reactivation were intermediate in their responding, being not significantly different from either the group that received vehicle at reactivation $(p=0.061)$ or the group that received vehicle at reactivation, but had the CS omitted at test $(p=0.063)$. All of the rats discriminated between the active and inactive levers (all $p$ values $<0.01$ ), indicating that performance of the instrumental response that had previously been reinforced with self-administered cocaine was not affected by the administration of MK-801 before memory reactivation. Rats in the CS-omission group also showed discrimination between the two levers (lever: $F_{(1,8)}=97.441, p<0.001$ ), further suggesting that in groups presented with the CS during test, the CS reinforced and enhanced the vigor of active lever pressing, although responding still occurred at lower rates in its absence.

Before testing and memory reactivation, all rats learned the instrumental response for intravenous cocaine during training (data not shown; lever: $F_{(1,39)}=166.12, p<0.001$; group: $F<1$; reactivation: $F<1$; group $\times$ reactivation: $F<1$ ). Similarly, all groups had equivalent exposure to the CS during training (data not shown, average CS presentations between 162.8 and 192.6; group: $F<1$; reactivation: $F<1$; group $\times$ reactivation: $F<1$ ).

As for experiment 1 , there were no differences between the groups in behavior during the reactivation session. Although MK-801 can produce the locomotor side effect of hyperactivity (Whishaw and Auer, 1989) this did not influence lever pressing during the memory reactivation session (data not shown; Lever: $F_{(1,24)}=76.77, p<0.001$; group: $\left.F<1\right)$. There were also no differences in CS exposure during the memory reactivation session (data not shown; group, $F_{(2,24)}=1.980$, n.s.).

\section{Discussion}

The data reported here demonstrate that activity at the NMDA subtype of glutamate receptor (NMDAR) in the BLA is necessary for drug-associated memory reconsolidation. Antagonism of NMDARs in conjunction with a memory reactivation session strongly attenuated the conditioned reinforcing properties of the previously drug-paired CS and so prevented it subsequently from supporting both new instrumental behavior, which can be considered a measure of flexible drug seeking (Di Ciano and Everitt, 2004) and the performance of a previously acquired instrumental drug-seeking response, which models an aspect of relapse behavior. The NMDAR dependence of the reconsolidation process was shown to be time-limited; NMDAR antagonism immediately after the memory reactivation session had no effect on memory reconsolidation as assessed by the ability of the CS to support the subsequent acquisition of a new instrumental response. Furthermore, we have demonstrated this amnestic effect after intra-BLA infusions of an NMDAR antagonist, providing clear evidence that the BLA is a primary locus of action for the effects of systemically administered NMDAR antagonists on CS-drug memory reconsolidation.

The specific targeting of the BLA in experiment 1 of this study also allows for direct comparison with studies of the genetic mechanisms of memory reconsolidation, because knockdown of the immediate early gene zif268 within the BLA also profoundly disrupts drug-associated memory reconsolidation (Lee et al., 2005, 2006a). Thus, both NMDAR antagonism and zif268 knockdown in conjunction with memory reactivation abolished the conditioned reinforcing properties of previously drug-paired CSs, subsequently impairing the acquisition of new drug-seeking behavior and attenuating previously learned instrumental responding for drug. Western blot analysis of Zif268 expression levels (experiment 1c) revealed that there was a downregulation of Zif268 expression when the NMDAR antagonist D-APV was administered intracerebrally immediately before memory retrieval, but not when D-APV was administered after memory retrieval, or in the absence of memory retrieval. These data strongly suggest that the effect of activity at NMDARs in the reconsolidation of drug-associated memories is mediated downstream by the plasticity-related immediate early gene zif 268 .

Our findings demonstrate that antagonism of NMDARs before memory retrieval specifically disrupted CS-drug memory reconsolidation, because administration of the NMDAR antagonist without the reactivation of the CS-drug memory had no effect on the capacity of the previously drug-paired CS to function as a conditioned reinforcer and support the acquisition of a new instrumental response. Indeed, responding for the previously drug-paired CS by animals infused, but not reactivated, increased over time, described previously as the "incubation of cocaine craving" by Grimm et al. (2001). A possible alternative account of the persistent impairment in instrumental responding shown by the experimental group receiving infusions of D-APV before memory reactivation, that the D-APV infusion induced an aversive state that acted to countercondition the CS, can be discounted, because no place aversion could be conditioned as a result of the intra-amygdala D-APV infusion.

In addition to our use of a behavioral procedure that isolates conditioned reinforcement and so models the acquisition of new drug-seeking responses that may greatly affect the propensity to relapse (Di Ciano and Everitt, 2004), in experiment 2 we used a more frequently studied and translationally relevant model of relapse to a previously acquired drug-seeking response (Lu et al., 
2005, 2006; Fuchs et al., 2006). Moreover, we studied the effects of a systemically administered NMDAR antagonist, MK-801, because systemic administration of pharmacological agents is likely to be of greater translational utility. The results of experiment 2 showed that it was possible to reduce the ability of a previously drugpaired CS to trigger and maintain instrumental responding previously reinforced by drug, through the disruption of the reconsolidation of CS-drug memories with MK-801. MK-801 administered before the memory reactivation session impaired subsequent lever pressing for the first 15 min of the subsequent test session, before the floor effect produced by withinsession extinction. This effect of MK-801 was reactivation-dependent and, moreover, the responding of the MK-801treated group did not differ from that of a control group in which the CS was omitted during test. Thus the reduction of drug seeking after MK-801 treatment was equivalent to omission of the CS altogether, suggesting that the treatment disrupted selectively the ability of the CS to influence drug-seeking responses. This effect was most clearly seen during the first 15 min of the test session, when cue-maintained drug seeking was highest in the vehicle group; it is possible that a larger dose of MK-801, or another NMDAR antagonist, may reduce this cue-maintained responding over a longer period of time. However, both the MK-801 and CS- omission groups continued to respond on the active lever, which is consistent with findings suggesting that instrumental memories apparently do not undergo a reconsolidation process (Hernandez and Kelley, 2004), and with our previous findings with knockdown of the plasticity-related immediate early gene zif268 in the amygdala (Lee et al., 2006a). Furthermore, in experiment 1 , there was no effect of NMDAR antagonism immediately before retrieval on subsequent nosepoking behavior, which had been reinforced previously with cocaine. This suggests that instrumental responding was not disrupted by the manipulations reported here, but rather that the effects of the drug-associated stimuli on behavior were attenuated, thus leading to a reduction in instrumental responding.

The impairment in CS-drug memory reconsolidation reported here is consistent with the finding that MK-801 can disrupt the reconsolidation of a cocaine-conditioned place preference (Kelley et al., 2007). However, the results of the present study provide an advance in at least two respects. First, because conditioned place preference (CPP) may be supported by a number of different associations and behavioral responses, including pavlovian conditioned approach, reward expectancy, and even conditioned reinforcement (Everitt and Robbins, 1992; Schechter and Calcagnetti, 1993), as well as both contextual and discrete cue influences (Ito et al., 2006), it is not clear what impairments in CPP reflect in psychological terms, nor how an impairment in CPP relates to the instrumental behaviors of drug seeking and drug taking. In contrast, experiment 1 specifically demonstrated that the CS-drug association was disrupted, because this is the only association that supports the acquisition of a new response for conditioned reinforcement (Mackintosh, 1974). Furthermore, the results demonstrated that even well established drug memories can be disrupted. In contrast to the CPP procedure, in which rats are typically administered three to four injections of an
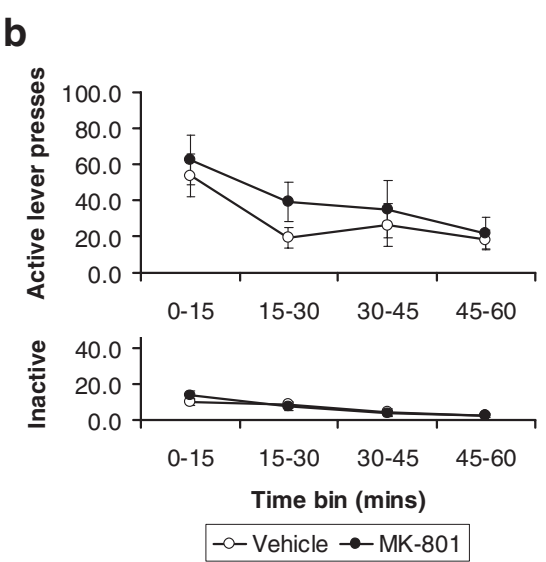

MK-801-- - - CS omission

Figure 6. Cue-induced relapse to cocaine seeking. $\boldsymbol{a}$, Lever pressing during test by rats that had received injections of MK- 801 or saline vehicle $30 \mathrm{~min}$ before the memory reactivation session. Inactive lever presses had no consequence; active lever presses ( $n=9$ per group). $\boldsymbol{b}$, Lever pressing during test by rats that received the injections without a memory reactivation session $(n=$ 6 per group). Data are presented as mean \pm SEM.

addictive drug (Kelley et al., 2007), we have shown here that CSdrug memory reconsolidation can be disrupted after the selfadministration of cocaine, and after extensive (180-300) CS-cocaine pairings. These data strongly suggest that the disruption of CS-drug memory reconsolidation with NMDAR antagonists in a realistic animal model of drug-seeking behavior is a feasible therapeutic strategy in human addicts trying to achieve abstinence.

Our data support the hypothesis that NMDARs within the BLA are necessary for the restabilization of drug-associated memories, because antagonism of these receptors prevented the memory from reconsolidating. The results are consistent, therefore, with our previous demonstration that systemic NMDAR antagonism with MK-801 disrupts CS-fear memory reconsolidation (Lee et al., 2006b) and cue-maintained sucrose seeking (Lee and Everitt, 2008). However, the present results and our previous work (Lee et al., 2006b) are in contrast to the finding that infusions of either D-APV or the NR2B-specific NMDAR antagonist ifenprodil before memory reactivation apparently prevent the destabilization of a CS-fear memory, protecting the "reactivated" memory from the amnestic effects of anisomycin (Ben Mamou et al., 2006). It is not yet clear whether these discrepant results reflect differences in the selective involvement of NMDAR subtypes in memory reconsolidation, procedural differences between the studies, or different cellular and molecular mechanisms underlying CS-drug and CS-fear memory reconsolidation.

Our data also differ from previous studies investigating the necessity of the NMDAR in memory reconsolidation with our finding that NMDAR antagonism was only sufficient to disrupt drugassociated memory reconsolidation if it was administered before, and not immediately after, the memory reactivation session. This contrasts with studies of the reconsolidation of object recognition memory (Akirav and Maroun, 2006) and odor-reward memory (Torras-Garcia et al., 2005), in which the central administration of the NMDAR antagonist APV immediately after the memory reactivation session was sufficient to produce subsequent memory impairments. These apparent temporal differences in NMDAR dependence may be related to the length of the reactivation sessions. The studies in which postreactivation NMDAR antagonism was found to disrupt memory reconsolidation used brief memory reactivation sessions [ $5 \mathrm{~min}$ and $90 \mathrm{~s}$, respectively, for Akirav and Maroun, (2006) and Torras-Garcia et al. (2005)] compared with experiment 1, in 
which the reactivation session lasted $15 \mathrm{~min}$. This implies that the hypothesized restabilization process mediated by NMDARs occurs rapidly after the memory has destabilized, suggesting that in the present study, the memory had already restabilized before the end of the reactivation session.

In conclusion, we have shown that rats that have previously learned to self-administer cocaine and have extensive CS-drugpairing experience can have their CS-maintained drug-seeking behavior reduced by undergoing a single treatment with an NMDAR antagonist, in conjunction with a drug-memory reactivation session, and that the effects of NMDAR antagonism are correlated with reductions in the downstream expression of the immediate early gene zif268. We have identified that NMDARs within the BLA, a site known to be involved with conditioned reinforcement, drug memory consolidation, and reconsolidation, are necessary for this effect, and that systemic administration of an NMDAR antagonist during memory reactivation is sufficient to produce a reduction in drugseeking behavior. Therefore, these results indicate that NMDAR antagonism may be a translationally relevant form of relapse prevention therapy in abstinent drug addicts.

\section{References}

Akirav I, Maroun M (2006) Ventromedial prefrontal cortex is obligatory for consolidation and reconsolidation of object recognition memory. Cereb Cortex 16:1759-1765.

Ben Mamou C, Gamache K, Nader K (2006) NMDA receptors are critical for unleashing consolidated auditory fear memories. Nat Neurosci 9:1237-1239.

Burns LH, Robbins TW, Everitt BJ (1993) Differential effects of excitotoxic lesions of the basolateral amygdala, ventral subiculum and medial prefrontal cortex on responding with conditioned reinforcement and locomotor activity potentiated by intra-accumbens infusions of D-amphetamine. Behav Brain Res 55:167-183.

Cador M, Robbins TW, Everitt BJ (1989) Involvement of the amygdala in stimulus-reward associations: interaction with the ventral striatum. Neuroscience 30:77-86.

Cardinal RN, Aitken MRF (2006) ANOVA for the Behavioural Sciences Researcher. London: Lawrence Erlbaum Associates.

Di Ciano P, Everitt BJ (2004) Conditioned reinforcing properties of stimuli paired with self-administered cocaine, heroin or sucrose: implications for the persistence of addictive behavior. Neuropharmacology 47:202-213.

Everitt BJ, Robbins TW (1992) Amygdala-ventral striatal interactions and reward-related processes. In: The amygdala: neurobiological aspects of emotion, memory, and mental dysfunction (Aggleton JP, ed), pp 401429. New York: Wiley.

Everitt BJ, Parkinson JA, Olmstead MC, Arroyo M, Robledo P, Robbins TW (1999) Associative processes in addiction and reward: the role of amygdala-ventral striatal subsystems. Ann N Y Acad Sci 877:412-438.

Everitt BJ, Dickinson A, Robbins TW (2001) The neuropsychological basis of addictive behaviour. Brain Res Rev 36:129-138.

Fuchs RA, Branham RK, See RE (2006) Different neural substrates mediate cocaine seeking after abstinence versus extinction training: a critical role for the dorsolateral caudate-putamen. J Neurosci 26:3584-3588.

Grimm JW, Hope BT, Wise RA, Shaham Y (2001) Incubation of cocaine craving after withdrawal. Nature 412:141-142.

Hellemans KG, Everitt BJ, Lee JL (2006) Disrupting reconsolidation of conditioned withdrawal memories in the basolateral amygdala reduces suppression of heroin seeking in rats. J Neurosci 26:12694-12699.

Hernandez PJ, Kelley AE (2004) Long-term memory for instrumental responses does not undergo protein synthesis-dependent reconsolidation upon retrieval. Learn Mem 11:748-754.

Ito R, Robbins TW, McNaughton BL, Everitt BJ (2006) Selective excitotoxic lesions of the hippocampus and basolateral amygdala have dissociable effects on appetitive cue and place conditioning based on path integration in a novel Y-maze procedure. Eur J Neurosci 23:3071-3080.

Kantak KM, Black Y, Valencia E, Green-Jordan K, Eichenbaum HB (2002) Dissociable effects of lidocaine inactivation of the rostral and caudal ba- solateral amygdala on the maintenance and reinstatement of cocaineseeking behavior in rats. J Neurosci 22:1126-1136.

Kelley JB, Anderson KL, Itzhak Y (2007) Long-term memory of cocaineassociated context: disruption and reinstatement. Neuroreport 18:777-780.

Lee JL, Everitt BJ (2008) Appetitive memory reconsolidation depends upon NMDA receptor-mediated neurotransmission. Neurobiol Learn Mem 90:147-154.

Lee JL, Everitt BJ, Thomas KL (2004) Independent cellular processes for hippocampal memory consolidation and reconsolidation. Science 304:839-843.

Lee JL, Di Ciano P, Thomas KL, Everitt BJ (2005) Disrupting reconsolidation of drug memories reduces cocaine seeking behavior. Neuron 47:795-801.

Lee JL, Milton AL, Everitt BJ (2006a) Cue-induced cocaine seeking and relapse are reduced by disruption of drug memory reconsolidation. J Neurosci 26:5881-5887.

Lee JL, Milton AL, Everitt BJ (2006b) Reconsolidation and extinction of conditioned fear: inhibition and potentiation. J Neurosci 26:10051-10056.

Lewis DJ (1979) Psychobiology of active and inactive memory. Psychol Bull 86:1054-1083.

Lu L, Hope BT, Dempsey J, Liu SY, Bossert JM, Shaham Y (2005) Central amygdala ERK signaling pathway is critical to incubation of cocaine craving. Nat Neurosci 8:212-219.

Lu L, Uejima JL, Gray SM, Bossert JM, Shaham Y (2006) Systemic and central amygdala injections of the $\mathrm{mGluR}_{2 / 3}$ agonist LY379268 attenuate the expression of incubation of cocaine craving. Biol Psychiatry 61:591-598.

Mackintosh NJ (1974) The psychology of animal learning. London: Academic.

Matus-Amat P, Higgins EA, Sprunger D, Wright-Hardesty K, Rudy JW (2007) The role of dorsal hippocampus and basolateral amygdala NMDA receptors in the acquisition and retrieval of context and contextual fear memories. Behav Neurosci 121:721-731.

Meil WM, See RE (1997) Lesions of the basolateral amygdala abolish the ability of drug associated cues to reinstate responding during withdrawal from self-administered cocaine. Behav Brain Res 87:139-148.

Mokin M, Keifer J (2005) Expression of the immediate-early gene-encoded protein Egr-1 (zif268) during in vitro classical conditioning. Learn Mem 12:144-149.

Nader K (2003) Memory traces unbound. Trends Neurosci 26:65-72.

O’Brien CP, Childress AR, McLellan AT, Ehrman R (1992) Classical conditioning in drug dependent humans. Ann N Y Acad Sci 654:400-415.

Parkinson JA, Roberts AC, Everitt BJ, Di Ciano P (2005) Acquisition of instrumental conditioned reinforcement is resistant to the devaluation of the unconditioned stimulus. Q J Exp Psychol B 58:19-30.

Przybyslawski J, Sara SJ (1997) Reconsolidation of memory after its reactivation. Behav Brain Res 84:241-246.

Robinson TE, Berridge KC (1993) The neural basis of drug craving: an incentive-sensitization theory of addiction. Brain Res Rev 18:247-291.

Sadler R, Herzig V, Schmidt WJ (2007) Repeated treatment with the NMDA antagonist MK-801 disrupts reconsolidation of memory for amphetamineconditioned place preference. Behav Pharmacol 18:699-703.

Schechter MD, Calcagnetti DJ (1993) Trends in place preference conditioning with a cross-indexed bibliography 1957-1991. Neurosci Biobehav Rev $17: 21-41$.

Stewart J, de Wit H, Eikelboom R (1984) Role of unconditioned and conditioned drug effects in the self-administration of opiates and stimulants. Psychol Rev 91:251-268.

Suzuki A, Josselyn SA, Frankland PW, Masushige S, Silva AJ, Kida S (2004) Memory reconsolidation and extinction have distinct temporal and biochemical signatures. J Neurosci 24:4787-4795.

Thomas KL, Arroyo M, Everitt BJ (2003) Induction of the learning and plasticity-associated gene Zif268 following exposure to a discrete cocaineassociated stimulus. Eur J Neurosci 17:1964-1972.

Torras-Garcia M, Lelong J, Tronel S, Sara SJ (2005) Reconsolidation after remembering an odor-reward association requires NMDA receptors. Learn Mem 12:18-22.

Whishaw IQ, Auer RN (1989) Immediate and long-lasting effects of MK801 on motor activity, spatial navigation in a swimming pool and EEG in the rat. Psychopharmacology 98:500-507. 